\title{
Electric dipoles and phase stability in nematic liquid crystals
}

\author{
By A. G. VANAKARAS and D. J. PHOTINOS \\ Department of Physics, University of Patras, Patras 26110, Greece
}

(Received 16 January 1995; accepted 22 March 1995)

\begin{abstract}
A theory for the nematic-isotropic $(\mathrm{N}-\mathrm{I})$ phase transition of prolate uniaxial molecules with longitudinal dipole moments is presented. The theory is based on the variational cluster expansion, truncated after the two-molecule term, and is implemented for polar hard spherocylinders with and without attractions, and for polar linear arrays of Lennard-Jones interaction centres. We find that the dipole interactions substantially shift the N-I transition temperature and strongly promote antiparallel molecular association, but have a weak effect on the order parameters, the pressure, and the $\mathrm{N}-\mathrm{I}$ coexistence densities. The effect of dipoles on phase stability is very sensitive to their position within the molecular frame. Off-centre dipoles are shown to give rise to phase re-entrance according to the sequence $\mathrm{N}-\mathrm{I}-\mathrm{N}$ on heating at constant density. The theory does not predict a stable ferroelectric nematic phase.
\end{abstract}

\section{Introduction}

The history of electric dipole interactions in molecular theories of liquid crystals dates back to the suggestion of Born [1] that the formation of liquid crystalline phases is a result of such interactions. Although it is now well established that the essential condition for liquid crystallinity is the anisotropy in the shape of the molecules [2] and not the presence of dipole moments, the latter are known to give rise to various phenomena in mesophases of compounds with strongly polar groups. Thus dipolar interactions have been attracting considerable attention over the last two decades in connection with ferroelectricity [3], smectic phase structure [4], molecular association [5, 6] and phase re-entrance [7] phenomena.

The role of dipolar interactions in the stabilization of mesophases has been the subject of recent theoretical investigations by analytic $[8,9]$ and computer simulation [10-14] methods. Such studies are motivated to a large extent by expectations $[15,16]$ that a polar (ferroelectric) nematic phase might be achievable. In spite of the valuable insights gained, especially from the simulations, the precise role of the dipolar interactions is not fully understood in general. The main reason for this is that the dipolar interaction always appears in combination with the basic shape anisotropy and possibly with other localized interactions. The reflection of such interplay of interactions on the physical properties of the phase is often rather sensitive to the details of the molecular structure [17]. For example, it was recently demonstrated $[18,19]$ that, depending on their position and orientation within the molecular frame, dipole moments can have a substantial influence or practically no effect at all on nematic ordering. To fully appreciate the effects that could be produced by polar groups it is therefore necessary to study the simultaneous dependence of the theoretical predictions on a number of molecular parameters. This scanning of phase behaviour vis a vis molecular parameterization is difficult to do by computer simulations since as a result of the long range of the dipolar interaction, such simulations 
are too time consuming to be carried out over the full field of the relevant parameters; furthermore, they are not free of ambiguities regarding the use of boundary conditions $[10,11,14]$. Analytic calculations, on the other hand, are based on statistical mechanical approximations but they are more efficient for a coarse scanning of the conditions under which the effects of dipolar interactions would be enhanced. Accordingly, the results of such calculations could guide experiments and computer simulations by narrowing down the field of molecular parameters over which interesting dipolar effects could be observed. It is in this spirit that we undertook the present study.

This paper is part of a more general investigation of dipolar interactions in liquid crystals. It addresses theoretically the influence of molecular polarity on the nematic-isotropic phase transition for the simplest and most common case of mesogens, namely, elongated uniaxial molecules. Our intention is to attempt a rationalization of the general characteristics of dipolar effects in these systems rather than to provide quantitatively accurate results. In accord with such an intention we have chosen to use an analytic approach based on the variational cluster expansion [20] of the free energy. This approach, compared to others that have been used to describe dipolar interactions in liquid crystals $[8,9,16,21,22]$, combines the following advantages:

(a) its accuracy is systematically upgradable by including contributions from higher-order clusters, while statistical mechanical consistency is maintained at each level of approximation;

(b) it is applicable to fluids of any symmetry as well as to solids;

(c) it allows the explicit modelling of intermolecular interactions to any detail (as opposed to the implicit introduction of interactions, for example, via correlation functions), and this modelling is not restricted by, or coupled in any way to, the level at which the statistical mechanical approximation is implemented.

The latter feature is particularly important since the manifestation of dipolar effects is in general sensitive to a number of molecular factors, aside from the magnitude of the dipole moment, and therefore such factors should not be restricted or distorted in order to comply with the statistical mechanical approximation employed (as is the case, for example, with lattice models [21] or with decoupling schemes $[16,23]$ ).

In the present calculations the variational cluster series is truncated past the two-molecule terms, thus providing the lowest order approximation that allows the description of phase transition properties from the explicit form of the intermolecular potential. Because of its more general applicability to liquid crystals, this procedure is described in some detail in section 2, where the free energy for a positionally uniform and anisotropic fluid is derived together with the expression for ensemble averages. In the case of hard particles without dipolar interactions the free energy reduces to that proposed by the Onsager theory for the $\mathrm{N}-\mathrm{I}$ phase transition $[24,25]$. The main body of our calculations concerns dipolar spherocylinders, a system that has recently been studied in some detail by Monte Carlo simulations $[12,13]$. The results are presented in section 3 . We have also done some calculations allowing for anisotropically distributed attractive forces and Lennard-Jones potentials, in order to see how the manifestations of the dipolar part of the interaction are influenced by the nature of the non-polar part. The results of these calculations are presented in section 4. A discussion of the limitations of the results and of 
their relevance to real liquid crystals is given in section 5 together with the conclusions.

\section{The nematic-isotropic transition in the variational cluster approximation}

The variational cluster expansion method has been applied in the past [26-28] to study certain simplified models of liquid crystals consisting of orientable particles with fixed positions on a regular lattice. The statistical mechanical framework of these studies was obtained by a straightforward extension of the work of Strieb et al. [20] on Heisenberg ferromagnets. The theory we introduce in this section is founded on the same methodology, but is differs from that of [26-28] in that it allows for full positional freedom of the molecules in the fluid phase.

The configurational partition function $Z$ for a system of $N$ rigid molecules in thermal equilibrium at temperature $T$ is given by

$$
Z=\left(\frac{1}{N !}\right) \int \mathrm{d}\{i\} \prod_{(i, j)} \exp [-\beta U(i, j)],
$$

where $\beta=1 / k T, U(i, j)$ is the interaction potential between a pair of molecules $(i, j)$ and $\{i\}$ represents collectively the configurational degrees of freedom (positional and orientational variables) of the $N$ molecules.

By introducing a variational field $\varphi(i)$ one can decompose the pair potential as follows:

$$
\beta U(i, j)=H_{0}(i, j)+H^{\prime}(i, j)
$$

where $H_{0}=\varphi(i)+\varphi(j)$ and $H^{\prime}(i, j)=\beta U(i, j)-\varphi(i)-\varphi(j)$.

One may then rewrite the partition function as

$$
Z=Z_{0}\left\langle\prod_{(i, j)} \exp \left[-H^{\prime}(i, j)\right]\right\rangle_{0}
$$

Here, $Z_{0}=(1 / N !) z_{0}^{N}$ is the reference partition function, with

$$
z_{0}=\int \mathrm{d}(1) \exp [-(N-1) \varphi(1)],
$$

and $\langle\ldots\rangle_{0}$ indicates averaging with respect to the variational interaction $H_{0}(i, j)$, namely

$$
\langle\ldots\rangle_{0}=\int \mathrm{d}\{i\}(\ldots) \prod_{i=1}^{N} p_{1}(i),
$$

where the single molecule probabilities are given by

$$
p_{1}(i)=\left(1 / z_{0}\right) \exp [-(N-1) \varphi(i)] .
$$

The free energy $F=-(1 / \beta) \ln (Z)$ is accordingly written as the sum of two terms, $F=F_{0}+F^{\prime}$. The reference term $F_{0}$ involves only the variational field,

The term

$$
F_{0}=(1 / \beta)\left[\ln N !-N \ln z_{0}\right] .
$$

$$
F^{\prime}=-(1 / \beta) \ln \left\langle\prod_{(i, j)} \exp \left[-H^{\prime}(i, j)\right]\right\rangle_{\mathrm{o}},
$$

involves both the variational field and the intermolecular potential. 
Since $Z$ and $F$ are obviously independent of the variational field, their functional derivatives with respect to $\varphi$ vanish to all orders. Briefly, the variational cluster method consists in approximating $F$ by using a truncated cluster expansion for $F^{\prime}$. The variational field is then determined by imposing the vanishing of the functional derivative of the approximated $F$ with respect to $\varphi$. Here we shall restrict our consideration to the lowest order approximation, obtained by truncating the cluster series after the two-molecule terms. It may be shown $[20,29]$ that this truncation is equivalent to affecting the following approximation:

$$
\ln \left\langle\prod_{(i, j)} \exp \left[-H^{\prime}(i, j)\right]\right\rangle_{0} \approx \sum_{(i, j)} \ln \left\langle\exp \left[-H^{\prime}(i, j)\right]\right\rangle_{0} .
$$

Applying equation (9) to the right-hand side (r.h.s.) of equation (8) we obtain in the case of a fluid the following expression for the approximate free energy:

$$
F^{(2)}=(1 / \beta)\left\{\ln N !-N \ln z_{0}-\frac{N(N-1)}{2} \ln \left\langle\exp \left[-H^{\prime}(i, j)\right]\right\rangle_{0}\right\} .
$$

To the same approximation the ensemble average of any quantity $X\{i\}$ is given by

$$
\langle X\rangle^{(2)}=\langle X\rangle_{0}+\sum_{(i, j)}\left\{\left\langle X \exp \left[-H^{\prime}(i, j)\right]\right\rangle_{0} /\left\langle\exp \left[-H^{\prime}(i, j)\right]\right\rangle_{0}-\langle X\rangle_{0}\right\} .
$$

Furthermore, the requirement of zero functional derivative $\delta F^{(2)} / \delta \varphi$ provides the following condition for the self consistent determination of the variational field (to within a trivial additive constant):

$$
\varphi(1)=-\ln \left\{z_{0}^{-1} \int \mathrm{d}(2) \exp [-(N-2) \varphi(2)-\beta U(1,2)]\right\},
$$

which shows explicitly the dependence of $\varphi$ on the intermolecular potential.

Equations (10)-(12) specify the statistical mechanical framework at the level of the two-molecule cluster approximation. We now specialize these equations to the treatment of uniform fluids exhibiting uniaxial orientational order and consisting of cylindrically symmetric molecules. The potential for a pair of such molecules is a function of the intermolecular vector $\mathbf{r}_{i j}$, and of the unit vectors $\mathbf{e}_{i}, \mathbf{e}_{j}$ denoting the orientations of the symmetry axes of the molecules. Also, in a spatially uniform phase the distribution function $p_{1}$ of equation (6) is purely orientational, i.e.,

$$
p_{1}(i)=f\left(\mathbf{e}_{i}\right) / V
$$

where $V$ is the volume of the system. It therefore follows that the relevant variational field $\varphi(i)$ depends only on the molecular orientation $\mathbf{e}_{i}$.

The connection of the variational field to the intermolecular potential is established according to equation (12) through the irreducible cluster integral

$$
q\left(\theta_{1,2}\right)=\int\left\{1-\exp \left[-\beta U\left(\mathbf{r}_{1,2}, \mathbf{e}_{1}, \mathbf{e}_{2}\right)\right]\right\} \mathrm{d} \mathbf{r}_{1,2} .
$$

This integral, being a function of the relative orientation $\theta_{1,2}$ of the two molecules, can be expanded in a Legendre series as

$$
q\left(\theta_{1,2}\right)=\sum_{L} q_{L} P_{L}\left(\cos \theta_{1,2}\right)
$$

For purely hard body potentials $q\left(\theta_{1,2}\right)$ is simply the excluded volume function. In 
general, if the potential $U$ vanishes sufficiently rapidly with increasing intermolecular separation (which is the case for the potentials to be considered in this study) $q\left(\theta_{1,2}\right)$ is of the order of a volume $v_{a}$ spanning a few molecular diameters. If we therefore expand the r.h.s. of equation (12) in powers of $q\left(\theta_{1,2}\right) / V$, all but the linear term will be negligible in the limit of macroscopic $V$ and $N$. Accordingly we obtain

$$
\varphi\left(\theta_{1}\right)=\bar{q}\left(\theta_{1}\right) / V,
$$

where $\theta_{1}$ is the angle of molecule 1 relative to the principal axis of the uniaxial phase (nematic director) and $\bar{q}\left(\theta_{1}\right)$ is the average of $q\left(\theta_{1,2}\right)$ over the orientations of molecule 2 , namely,

$$
\bar{q}\left(\theta_{1}\right)=\int q\left(\theta_{1,2}\right) f\left(\theta_{2}\right) \mathrm{d} \cos \theta_{2}=\sum_{L} q_{L}\left\langle P_{L}\right\rangle P_{L}\left(\cos \theta_{1}\right) .
$$

The order parameters $\left\langle P_{L}\right\rangle$ are defined as

$$
\left\langle P_{L}\right\rangle=\int P_{L}(\cos \theta) f(\theta) \mathrm{d} \cos \theta
$$

and the orientational distribution $f(\theta)$ is given by

$$
f(\theta)=\exp [-\rho \bar{q}(\theta)] / \zeta
$$

Here, $\rho=N / V$ is the particle number density, and $\zeta$ is the normalization factor

$$
\zeta=\int \exp [-\rho \bar{q}(\theta)] \mathrm{d} \cos \theta
$$

The combination of equations (18) and (19) constitutes a set of self consistency conditions from which the order parameters $\left\langle P_{L}\right\rangle$ are determined given the set of coefficients $q_{L}$.

In view of equations $(16)-(20)$ (and using $\ln (N !) \approx N \ln (N)-N)$ equation (10) leads to the following expression for the free energy of a uniaxial fluid in the second order cluster approximation

$$
(\beta / N) F^{(2)}=\ln \rho-1-\ln \zeta-(\rho / 2) \sum_{L} q_{L}\left\langle P_{L}\right\rangle^{2}
$$

This expression can be made identical to the familiar Onsager free energy $[24,25]$ on noting from equations (17), (19), and (20) that $\ln \zeta+(\rho / 2) \sum_{L} q_{L}\left\langle P_{L}\right\rangle^{2}$ is equivalent to $-\int f(\theta) \ln f(\theta) \mathrm{d} \cos \theta-(\rho / 2) \int f\left(\theta_{1}\right) f\left(\theta_{2}\right) \mathrm{d} \cos \theta_{1} \mathrm{~d} \cos \theta_{2} \int \mathrm{d} \mathbf{r}_{1,2}\{1-$ $\exp [-\beta U(1,2)]\}$. Thus the cluster expansion provides yet another derivation of the Onsager result [24] (see also [25,30]), but in addition it offers a consistent way of treating statistical averages with equation (11).

From the free energy in equation (21) it is straightforward to obtain all the thermodynamic quantities of interest to the study of the nematic-isotropic phase transition. In particular, the configurational internal energy $E$, the pressure $P$, and the chemical potential $\mu$ are given by

$$
E /(N k T)=(\rho / 2) \beta \sum_{L} q_{L}^{\prime}\left\langle P_{L}\right\rangle^{2},
$$

where $q_{L}^{\prime}=\mathrm{d} / \mathrm{d} \beta q_{L}$,

$$
P /(\rho k T)=1+(\rho / 2) \sum_{L} q_{L}\left\langle P_{L}\right\rangle^{2},
$$

and

$$
\mu / k T=\ln \rho-\ln \zeta .
$$


The respective expressions for the isotropic phase are obtained by setting $\left\langle P_{L}\right\rangle=0$ for $L>0$ in equations (22-24). Accordingly the conditions of equal pressure and chemical potential for nematic-isotropic $(\mathrm{N}-\mathrm{I})$ phase coexistence yield the equations

and

$$
\rho_{\mathrm{I}} / \rho_{\mathrm{N}}=(2 / \zeta) \exp \left(-q_{0} \rho_{\mathrm{I}}\right)
$$

$$
\rho_{\mathrm{N}}+(1 / 2) \rho_{\mathrm{N}}^{2} \sum_{L} q_{L}\left\langle P_{L}\right\rangle^{2}=\rho_{\mathrm{I}}+\rho_{\mathrm{I}}^{2} q_{\mathrm{o}} / 2
$$

Similarly, the entropy change associated with the N-I transition at constant density $\rho$ is given by

$$
\Delta S_{\mathrm{N}-\mathrm{I}} /(N k)=-(\rho / 2) \beta \sum_{L>0} q_{L}^{\prime}\left\langle P_{L}\right\rangle^{2}
$$

The expressions for the thermodynamic quantities in equations (22-27) as well as the self-consistency conditions of equations $(18,19)$ are formally analogous to those derived in the context of the usual mean-field approximation (MFA) [31, 32]. The important difference is, however, that in the MFA the coefficients $q_{L}$ are essentially treated as input parameters whereas in the cluster approximation they are derived from the intermolecular potential, and therefore they bare explicitly the dependence on the molecular parameters and the temperature. A further important difference concerns the calculation of intermolecular averages where the correlation sum of equation (11) vanishes in the MFA.

\section{Nematic ordering of hard dipolar spherocylinders}

In this section we use the free energy in equation (21) to study the nematic ordering of hard spherocylinders possessing longitudinal electric dipole moments. The latter are assumed to be positioned along the axis of the spherocylinder at a distance $s$ from its centre. The molecular model is specified by four quantities, namely the diameter $D$ of the spherocylinder, the length $L$ of its cylindrical part, the magnitude $d$ of the dipole moment and the dipolar eccentricity $x$ which we define as $x=2 s / L$. The volume of the spherocylinder is denoted by $v_{0}$.

The essential geometrical parameter describing the orientability of the spherocylinder is the aspect ratio $L / D$. It is known from the Onsager theory that the accuracy of the approximate free energy in equation (21) rapidly deteriorates for small $L / D$. On the other hand, the aspect ratio of common nematogens is not much larger than 3 . In our calculations we considered aspect ratios from 3 to 7 . The results we report in this paper are for $L / D=5$, but they are representative of the qualitative trends obtained with other values of $L / D$ in the range mentioned earlier. The dipolar eccentricity $x$ was varied from 0 (corresponding to centrally positioned dipole moments) to 1 (dipoles positioned at the end of the cylindrical part of the spherocylinder) in order to study the dependence of the nematic properties on the position of the dipole along the molecular axis.

The intermolecular potential of the dipolar spherocylinders has an infinite steric part and a dipole-dipole interaction part. Accordingly the Boltzmann factor $\exp [-\beta U(1,2)]$ vanishes when the pair of spherocylinders overlap, and is equal to $\exp \left[-\beta_{\mathrm{d}} u_{\mathrm{dd}}(1,2)\right]$ otherwise. Here, $u_{\mathrm{dd}}(1,2)=\left[\left(\mathbf{e}_{1} \cdot \mathbf{e}_{2}\right)-3\left(\mathbf{r} \cdot \mathbf{e}_{1}\right)\left(\mathbf{r} \cdot \mathbf{e}_{2}\right)\right] /(r / D)^{3}$ is the dimensionless potential describing the interaction of the electric dipoles, and $\beta_{\mathrm{d}}=\beta d^{2} / D^{3}$ is a dimensionless parameter which may be regarded either as a reduced 
inverse temperature (for fixed dipole moment strength $d$ ), or as an effective coupling constant for the dipolar interactions. For polar mesogens of practical interest $\beta_{\mathrm{d}}$ is on the order of one. Most of our results refer to the range $0 \leqslant \beta_{\mathrm{d}} \leqslant 2$ which is fairly typical and illustrates sufficiently the essential trends. It should be pointed out, however, that due to the omission of higher order clusters the entire approximation grows unreliable for large $\beta_{\mathrm{d}}$.

Given the detailed form of the potential we first evaluate the expansion coefficients $q_{L}$ of the cluster integral in equation (14). The magnitude of these coefficients rapidly decreases with ascending $L$. The most significant $q_{L}$ 's are plotted as functions of $\beta_{\mathrm{d}}$ in figure 1 . The magnitude of the odd coefficients $q_{L}$ increases monotonically with $\beta_{\mathrm{d}}$ but remains substantially smaller than the magnitude of the next even coefficients
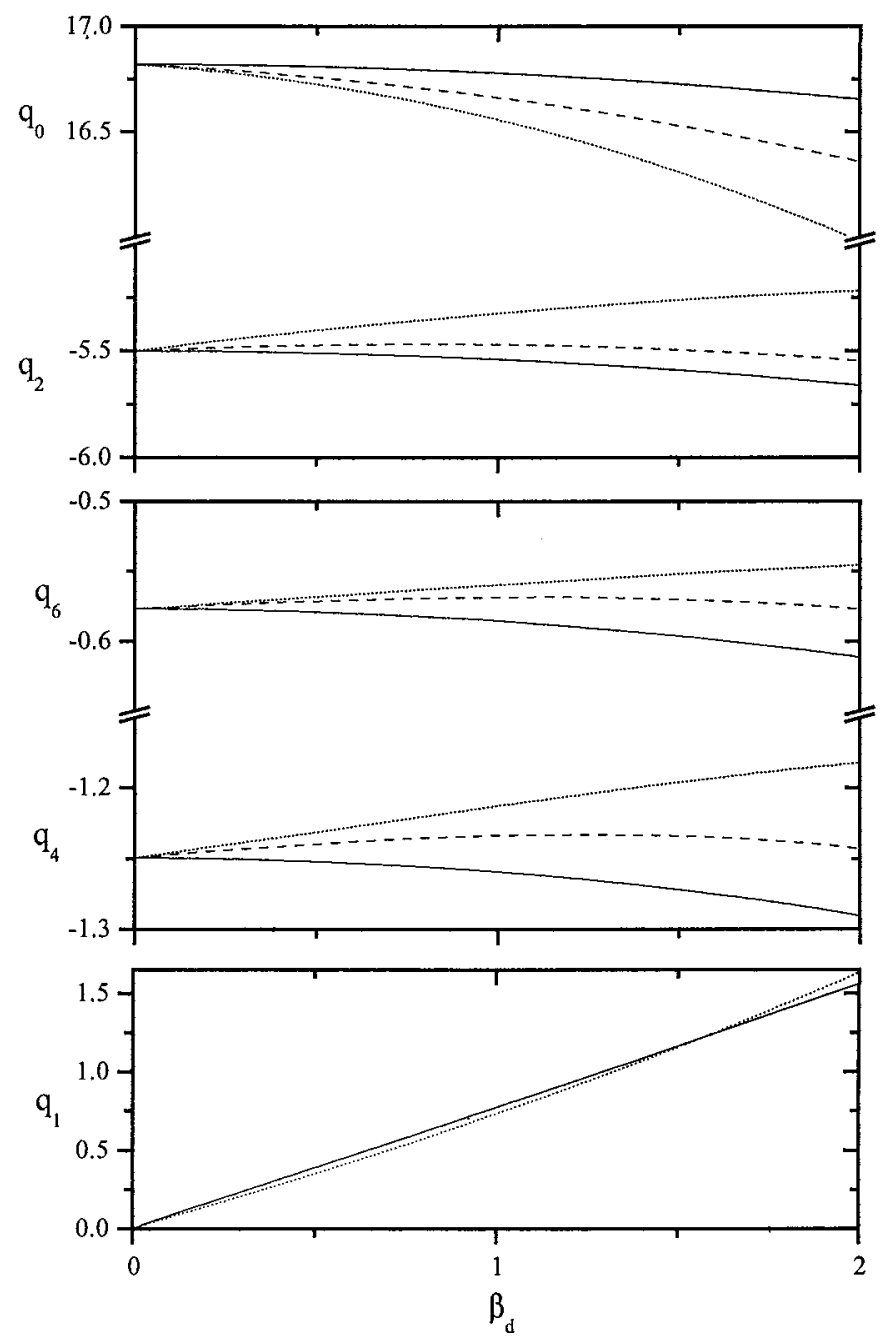

Figure 1. Expansion coefficients of equation (15) for dipolar spherocylinders as a function of the effective dipole coupling constant $\beta_{\mathrm{d}}$, and for three different values of the dipolar eccentricity parameter: $x=0$ for solid curves, $x=0.8$ for dashed curves and $x=1$ for dotted curves. The values of the coefficients are expressed in units of the volume $v_{0}$ of the spherocylinder and refer to $L / D=5$. 
$q_{L+1}$. For example at $\beta_{\mathrm{d}}=2, q_{3}$ and $q_{5}$ are smaller than $q_{6}$ by one order of magnitude. The numerical values of the even coefficients $q_{0}, q_{2}$, and $q_{4}$ for extremely off-centre dipoles $(x=1)$ do not differ greatly from those for central dipoles $(x=0)$. It is clear, however, from the plots in figure 1 that the trend of variation of these coefficients with $\beta_{\mathrm{d}}$ is sensitive to the eccentricity $x$. As will become evident later, this sensitivity of the trends has profound qualitative implications on phase stability.

The expansion coefficients are then used to evaluate the order parameters $\left\langle P_{L}\right\rangle$ from the self-consistency equations (18). The coefficients of rank $L>6$ are neglected in these calculations. The order parameters are, in turn, used in equations $(25,26)$ to detect phase coexistence. Only two stable phases, isotropic (I) and non-polar nematic $(\mathrm{N})$, are consistent with the free energy $F^{(2)}$ of equation (21). Neither a vapour-liquid coexistence nor a polar nematic phase is sustained by $F^{(2)}$ regardless of the details of the intermolecular potential. In particular, the lack of a liquid-vapour transition is not a peculiarity of the polar spherocylinder model; the conditions of equations $(25,26)$ do not produce coexisting isotropic phases of different densities for any value of $q_{0}$, i.e., for any form of the intermolecular potential and any value of the coupling constants. This is one of the several limitations associated with the neglect of larger cluster contributions to the free energy.

Returning to the $\mathrm{N}-\mathrm{I}$ phase transitions, we present the results for the pressure $P_{\mathrm{N}-\mathrm{I}}$, the nematic density $\rho_{\mathrm{N}}$, and the relative density difference $\left(\rho_{\mathrm{N}}-\rho_{1}\right) / \rho_{\mathrm{N}}$ at phase coexistence as functions of $\beta_{\mathrm{d}}$ and for various dipolar eccentricities in figure 2 . Except for the case of extreme eccentricity $(x=1)$, the variation of all three quantities over the entire range of $\beta_{\mathrm{d}}$ does not exceed a few per cent, indicating that the dipolar interaction has a limited effect on the phase equilbria. All the coexistence curves originate from a common value at $\beta_{\mathrm{d}}=0$ (where obviously the eccentricity is irrelevant), but exhibit different trends depending on $x$. To appreciate the implications of these curves it is useful to think of $\beta_{\mathrm{d}}$ as a measure of the dipole strength at fixed temperature. Figure $2(a)$ and $(b)$ then indicate that, depending on the eccentricity, increasing the magnitude of the dipole moment may raise or lower the coexistence pressure $P_{\mathrm{N}-\mathrm{I}}$ and the density $\rho_{\mathrm{N}}$ relative to their values in the absence of dipolar interactions $\left(\beta_{\mathrm{d}}=0\right)$. In particular, a small off-centre dipole is predicted to elevate the nematic density threshold (and the coexistence pressure) whereas a large off-centre dipole is predicted to lower it. A centrally positioned dipole always lowers the nematic density threshold (and coexistence pressure). Although our main concern is with relative changes caused by dipole interactions, it should be noted that $P_{\mathrm{N}-\mathrm{I}}$ and $\rho_{\mathrm{N}}$ at $\beta_{\mathrm{d}}=0$ being the coexistence pressure and nematic density of the Onsager theory for spherocylinders, are substantially overestimated with respect to values obtained from computer simulations [25].

In contrast to the athermal behaviour of purely hard body interactions, the inclusion of the dipolar component introduces a non-trivial dependence of the phase equilibria on the temperature. As a result, phase transitions become possible by varying the temperature at constant density. The transitions are first order. The value of $\beta_{\mathrm{d}}$ at which the $\mathrm{N}$-I phase transition occurs for a given density $\rho$ is determined from the condition $F_{\mathrm{N}}^{(2)}=F_{\mathrm{I}}^{(2)}$. The reduced inverse temperature at the transition $\left(\beta_{\mathrm{d}}\right)_{\mathrm{N}-\mathrm{I}}$ is plotted in figure $3(a)$ as a function of the density for various eccentricities. As expected all the curves originate from the same density value $\rho_{0}$ at high temperatures $\left(\beta_{\mathrm{d}}=0\right)$ where the system becomes athermal. The entropy change $(\Delta S)_{\mathrm{N}-\mathrm{I}}$ associated with the $\mathrm{N}-\mathrm{I}$ transition is plotted in figure $3(b)$ as a function of $\left(\beta_{\mathrm{d}}\right)_{\mathrm{N}-\mathrm{I}}$. Evidently $(\Delta S)_{\mathrm{N}-\mathrm{I}}$ increases rapidly with $\left(\beta_{\mathrm{d}}\right)_{\mathrm{N}-\mathrm{I}}$ but is not very sensitive to 

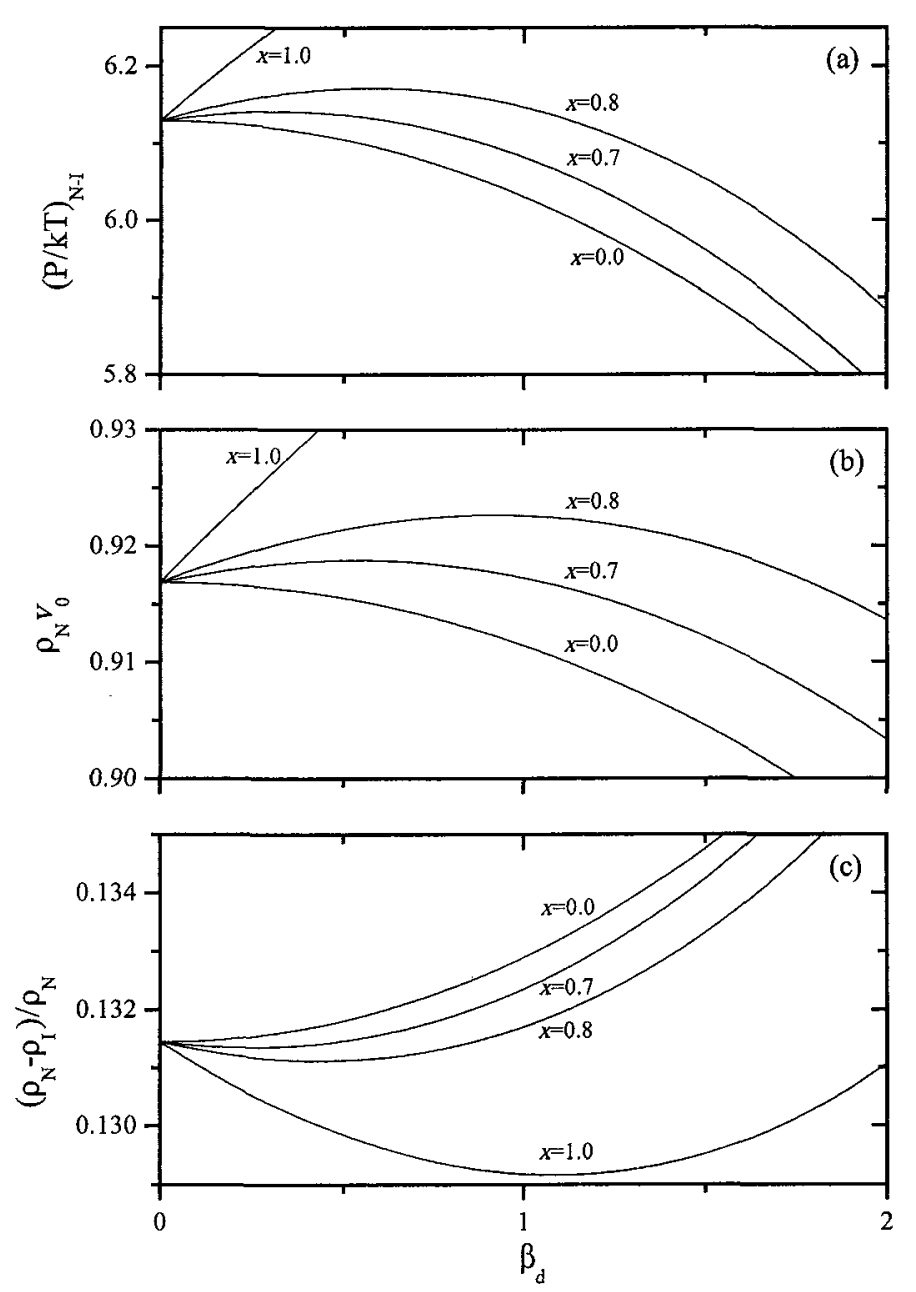

Figure 2. Nematic-isotropic coexistence of dipolar spherocylinders with $L / D=5$. Plots of the coexistence pressure $P_{\mathrm{N}-\mathrm{I}}$ (in units of $k T$ ), of the density of the nematic phase at coexistence $\rho_{N}$ (expressed as packing fraction in terms of the spherocylinder volume $v_{0}$ ) and of the relative density difference at coexistence $\left(\rho_{\mathrm{N}-} \rho_{\mathrm{I}}\right) / \rho_{\mathrm{N}}$ as a function $\beta_{\mathrm{d}}$ for various values of the eccentricity parameter $x$.

the eccentricity. Finally, the value of the principal order parameter $\left\langle P_{2}\right\rangle$ at the $\mathrm{N}$-I transition varies very little with $\left(\beta_{\mathrm{d}}\right)_{\mathrm{N}-\mathrm{I}}$ and $x\left(\left\langle P_{2}\right\rangle_{\mathrm{N}-\mathrm{I}}=0.831 \pm 0.001\right.$ for $0 \leqslant\left(\beta_{\mathrm{d}}\right)_{\mathrm{N}-\mathrm{I}} \leqslant 2$ and $\left.0 \leqslant x \leqslant 1\right)$.

The essential implication of the phase diagrams in figure $3(a)$ is that the temperature of the $\mathrm{N}-\mathrm{I}$ transition is strongly affected by the eccentricity $x$. It is clear that at densities $\rho<\rho_{0}$ the $\mathrm{N}-\mathrm{I}$ transition temperature for central dipoles is much higher than for off-centre dipoles of the same magnitude. A notable feature is that for large eccentricity $\left(\beta_{\mathrm{d}}\right)_{\mathrm{N}-\mathrm{I}}$ becomes a double valued function of $\rho$ in the density region $\rho>\rho_{0}$. This allows for the re-entrance sequence $\mathrm{N}-\mathrm{I}-\mathrm{N}$ by increasing the temperature at constant density. As implied by the example on the $x=0.8$ phase diagram of figure $3(a)$, off-centre dipoles can stabilize the isotropic phase (over a bounded temperature range $\beta_{-}<\beta_{\mathrm{d}}<\beta_{+}$) at densities $\rho>\rho_{0}$ that are well within the nematic regime of 

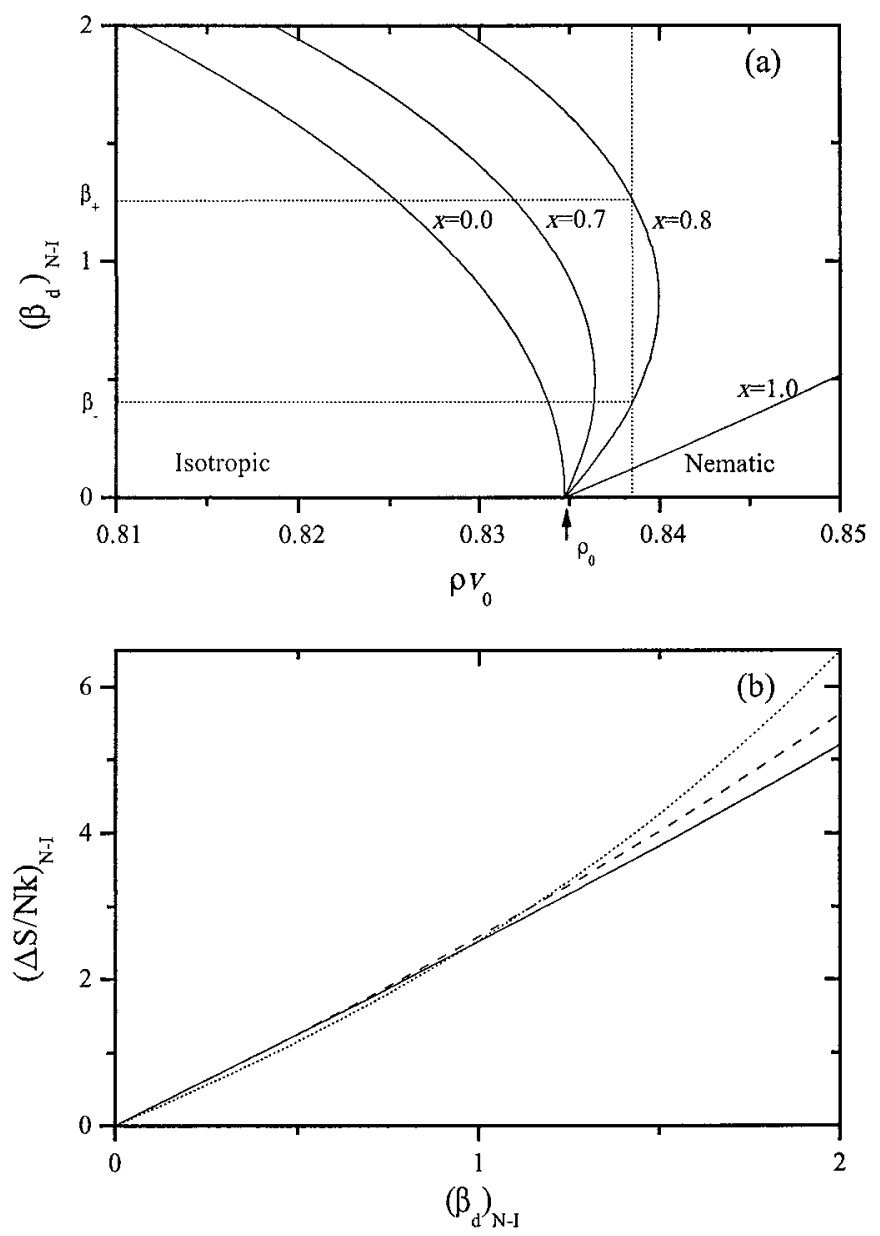

Figure 3. Nematic-isotropic phase transition at constant density for dipolar spherocylinders with $L / D=5$ and various dipolar eccentricity parameters $x$. (a) Plot of the reduced inverse temperature $\left(\beta_{\mathrm{d}}\right)_{\mathrm{N}-1}$ of the transition as a function of the density $\rho$ (expressed in units of the spherocylinder volume $\left.v_{0}\right)$. (b) Entropy change $\Delta S_{\mathrm{N}-\mathrm{I}}$ at the transition as a function of $\left(\beta_{\mathrm{d}}\right)_{\mathrm{N}-\mathrm{r}}$. The solid curve corresponds to eccentricity $x=0$, the dashed to $x=0.8$ and the dotted to $x=1$.

central dipoles (compare with the $x=0$ phase diagram). A similar N-I-N re-entrance sequence was recently predicted [33] for hydrogen-bonding hard spherocylinders.

Our calculations revealed that certain physical properties of the nematic phase are more sensitive to the dipolar eccentricity than others. For example the compressibility $(P / \rho k T)$ shows a variation of no more than $5 \%$ over the entire range of $x$ (and $\beta_{\mathrm{d}}$ ) for densities in the nematic as well as in the isotropic regions. Further examples are illustrated in figure 4 where the $\beta_{\mathrm{d}}$ dependence (at constant density $\rho v_{0}=0.8385$ ) of the order parameter $\left\langle P_{2}\right\rangle$, the configurational energy $E$ and the dipole correlation factors (Kirkwood $g$ factors) $[32,34]$ are plotted for central $(x=0)$ and off-centre $(x=0 \cdot 8)$ dipoles.

As the plots of figure 4(a) indicate, the two nematic phases produced by the molecules with $x=0.8$ and the nematic phase of the molecules with $x=0$ do not differ very much in their numerical values of $\left\langle P_{2}\right\rangle$. However, the high-temperature 

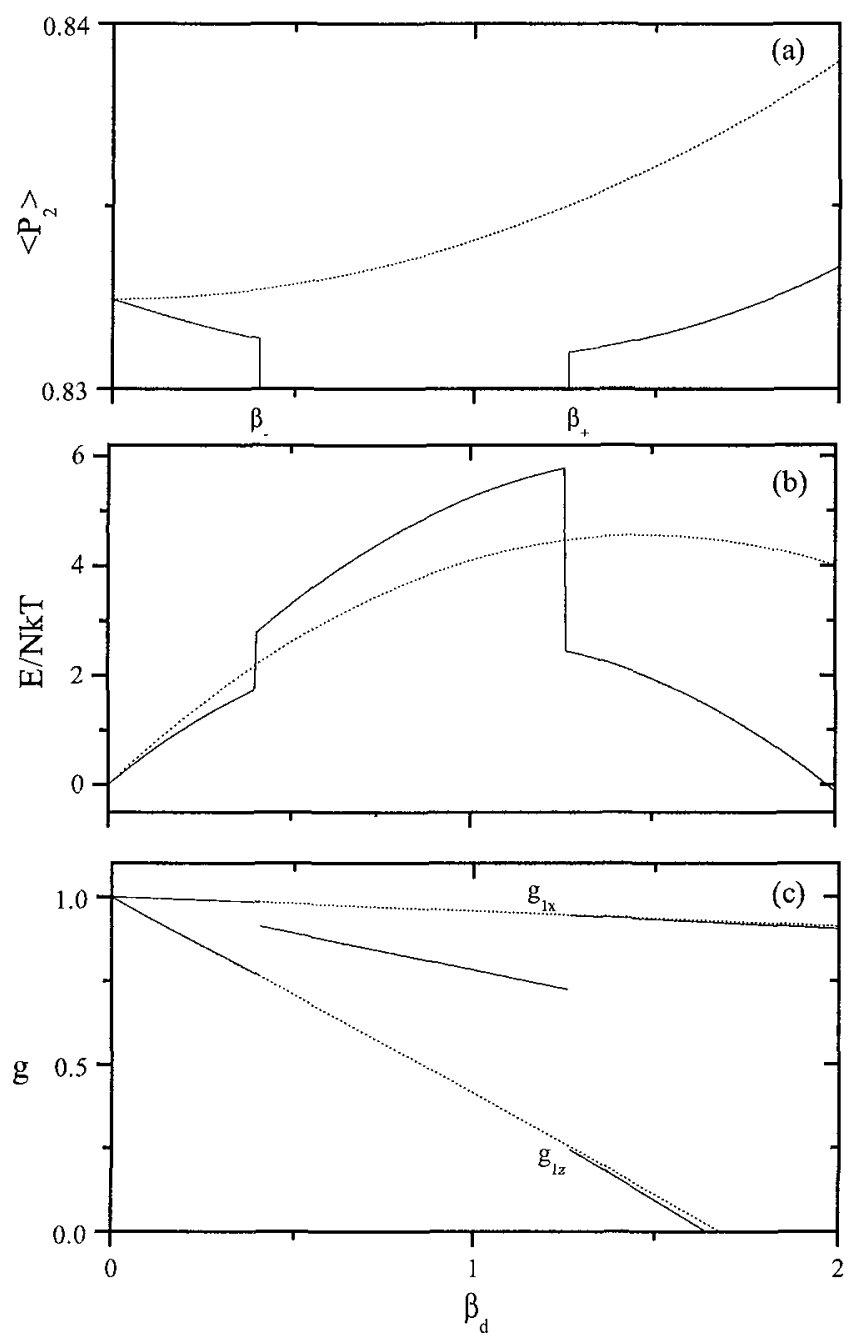

Figure 4. Plots of the order parameter $\left\langle P_{2}\right\rangle$, the internal configurational energy $E$ per molecule (expressed in units of $k T$ ), and Kirkwood dipole correlation factors $g_{z}, g_{x}$ (parallel and perpendicular to the nematic director) as a function of $\beta_{\mathrm{d}}$ for dipolar spherocylinders at packing fraction $\rho v_{0}=0.8385$. The dotted curves correspond to central dipoles, and the solid curves correspond to eccentricity $x=0 \cdot 8$.

nematic phase of the $x=0.8$ system exhibits an anomalous variation of the order parameter with $\beta_{\mathrm{d}}$, i.e., a decrease of the ordering with decreasing temperature. The normal trend is restored in the low-temperature nematic phase. A relatively high value (about $60 \%$ of $\left\langle P_{2}\right\rangle$ ) is predicted for the order parameter $\left\langle P_{4}\right\rangle$. Its variation with $\beta_{\mathrm{d}}$ follows that of $\left\langle P_{2}\right\rangle$ for both cases of eccentricity.

The plots of figure 4 (b) show that the internal configurational energy $E$, calculated from equation (22), varies considerably with $x$ at large $\beta_{\mathrm{d}}$. For hard dipolar bodies, $E$ is simply the average energy of the dipole-dipole interaction. Accordingly figure 4 (b) indicates that small dipoles increase the average dipolar energy of the system, irrespective of their position along the molecular axis, and that off-centre dipoles lead to lower dipolar energy than central ones in the nematic phase. 
The Kirkwood $g$ factors are given by

$$
g_{z}=1+(N-1)\left\langle\left(\mathbf{e}_{1} \cdot \mathbf{z}\right)\left(\mathbf{e}_{2} \cdot \mathbf{z}\right)\right\rangle /\left\langle\left(\mathbf{e}_{1} \cdot \mathbf{z}\right)^{2}\right\rangle .
$$

and similarly for $g_{x}$, where the intermolecular averages are evaluated according to equation (11), and the $\mathbf{z}$ axis is identified with the nematic director. The plots of the $g$ factors in figure $4(c)$ show that there is strong antiparallel $(g<1)$ association of the molecular dipoles. Furthermore, there is very little difference between $x=0$ and $x=0.8$, indicating that the position of the dipole moment along the molecular axis has minor effects on molecular association in the nematic phase. It is obvious from the negative values of $g_{z}$ at large $\beta_{\mathrm{d}}$ that the use of equation (11) leads to a gross overestimate of orientational correlations along the nematic director. This fact has been pointed out in [13] where the $g$ factors, together with other relevant quantities have been evaluated by Monte Carlo (MC) simulation for central dipoles, and for selected densities and dipole moment strength. The MC value for $g_{z}$ is, of course, positive and substantially smaller than 1 . The values of the transverse factor $g_{x}$ are in reasonable quantitative agreement with the $\mathrm{MC}$ results. Extensive quantitative comparisons of our results (for $x=0$ ) with the $\mathrm{MC}$ results [13] can not be made because of the large difference in the predicted nematic density threshold. A comparison restricted to the density $\rho v_{0}=0.356$ and $\beta_{\mathrm{d}}=6.0$, where both calculations predict an isotropic phase, shows that the dipolar energy is overestimated with respect to the MC results by roughly a factor of 2 , and similarly for the compressibility. The value of the isotropic $g$ factor $(0 \cdot 30)$ is appreciably smaller than the MC value $(0.41)$. Finally, a noticeable qualitative difference is in the distribution function $f(\theta)$ which according to our results has its maximum at $\theta=0$, whereas the $\mathrm{MC}$ results suggest an unusual behaviour at small angles with the maximum shifted to $\theta \approx 7^{\circ}$.

\section{The inclusion of attractions and soft repulsions}

The athermal structure of the phase diagrams in figures $2(b)$ and $3(a)$ near $\beta_{\mathrm{d}}=0$ reflects the use of hard body potentials to represent the non-polar part of the intermolecular interaction. It is therefore meaningful to ask how these diagrams would be affected if the restriction to purely hard body potential were relaxed. To address this question we have included attractions and soft repulsions in our calculations simply by introducing along the molecular axis a number $n_{\mathrm{s}}$ of evenly spaced identical interaction centres. The potential between an intermolecular pair of centres is taken to be isotropic. The total interaction potential for a pair of molecules is anisotropic by virtue of the linear arrangement of the interaction centres on each of the molecules. This is obviously just one of the many ways to depart from the hard spherocylinder potential, but it adequately represents the general implications of the inclusion of soft components in the non-polar part of the intermolecular potential. In the remaining of this section we present the diagrams of constant density phase transitions for two specific models of multicentre potentials.

\subsection{Hard spherocylinders with distributed attraction centres}

In this model the hard spherocylinder carries, in addition to the dipole moment, nine $\left(n_{\mathrm{s}}=9\right)$ centres of intermolecular attraction symmetrically positioned along its axis with uniform spacing $L /\left(n_{\mathrm{s}}-1\right)$. The potential of an intermolecular pair of 


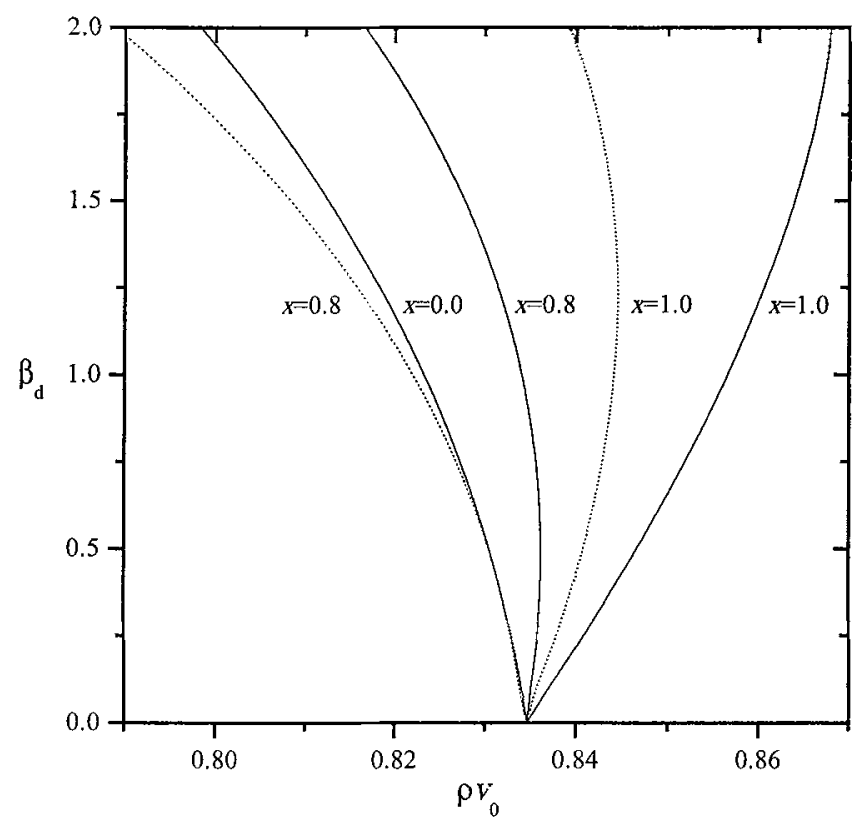

Figure 5. Constant density nematic-isotropic phase transition of dipolar spherocylinders $(L / D=5)$ with attractive interactions as in equation (29) with $n_{\mathrm{s}}=9$. Relative strength of attractions: $\beta_{\mathrm{a}} / \beta_{\mathrm{d}}=0.8$ for solid curves and 2.4 for dotted curves.

attraction centres is taken to vary as the inverse sixth power of their distance $r_{c c}$. Accordingly the total attractive potential for a pair of molecules is parameterized as follows:

$$
u_{\mathrm{a}} / k T=-\beta_{\mathrm{a}} / n_{\mathrm{s}}^{2} \sum_{\left(c, c^{\prime}\right)}\left(D / r_{c c^{\prime}}\right)^{6}
$$

where the summation extends over all combinations of intermolecular pairs of centres, and $\beta_{\mathrm{a}}$ measures the effective strength of the attractive interaction.

The results of the calculation for the phase transition at constant density using the above interaction are plotted in figure 5. They were obtained for two values of the ratio $\beta_{\mathrm{a}} / \beta_{\mathrm{d}}$ of attractive to dipolar interactions namely $\beta_{\mathrm{a}} / \beta_{\mathrm{d}}=0.8$ and $2 \cdot 4$. Inspection of these plots and comparison with figure $3(a)$, shows that the main effect of attractions is a more rapid reduction of the density of the $\mathrm{N}-\mathrm{I}$ transition with increasing $\beta_{\mathrm{d}}$. We note that as a result of this reduction $\beta_{\mathrm{d}}$ for $x=0.8$ becomes single valued in the curve with $\beta_{\mathrm{a}} / \beta_{\mathrm{d}}=2 \cdot 4$. In this case, the attractions are strong enough to completely mask the tendency of off-centre dipoles to produce nematic phase re-entrance. At high temperatures the interactions are dominated by the hardspherocylinder repulsions, and therefore the athermal behaviour of the system forces all the curves to originate from a common value of the density at $\beta_{\mathrm{d}}=0$.

\subsection{Linear arrays of Lennard-Jones interaction centres}

In this model we introduce soft repulsions as well as attractions by replacing the spherocylinder with a linear collection of evenly spaced Lennard-Jones (L-J) interaction centres. The molecular length $L$ is represented by the distance between 


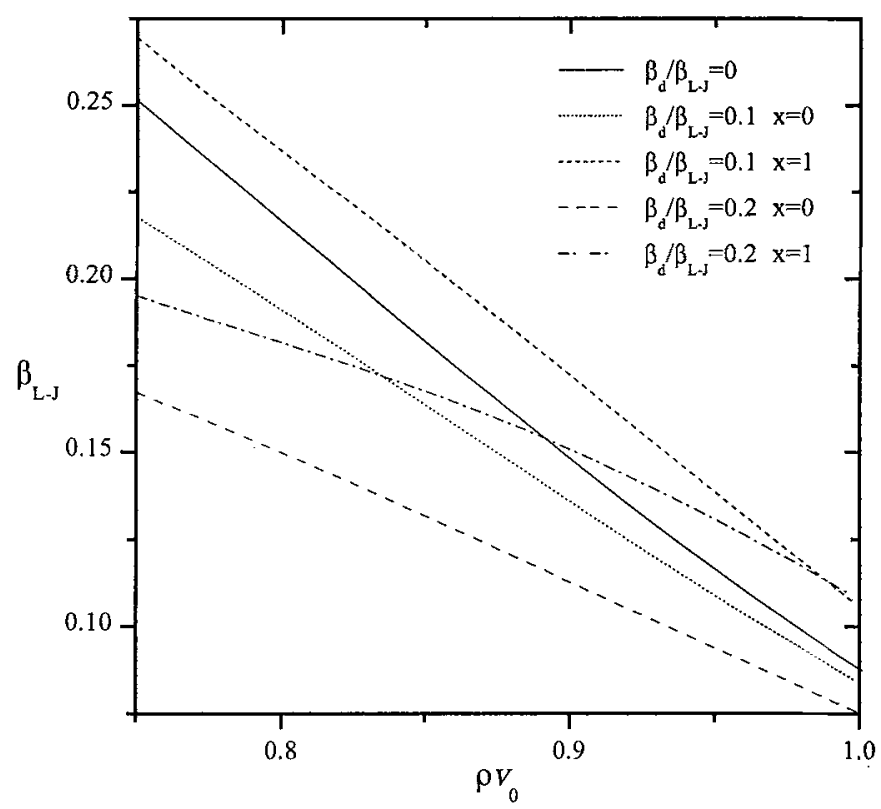

Figure 6. Constant density nematic-isotropic phase transition of L-J arrays with nine interaction centres, $L / D=5$, and a dipole moment along the axis of the array for various combinations of the relative strength $\beta_{\mathrm{d}} / \beta_{\mathrm{L}-\mathrm{s}}$ of dipolar to L-J interactions, and of the dipolar eccentricity parameter $x$.

the first and the last interaction centre of the array. The effective molecular diameter $D$ corresponds to the distance at which the L-J potential vanishes. The total L-J interaction for a pair of molecules is parameterized as follows:

$$
u_{\mathrm{L}-\mathrm{J}} / k T=\left(\beta_{\mathrm{L}-\mathrm{J}} / n_{\mathrm{s}}^{2}\right) \sum_{\left(c, c^{\prime}\right)}\left[\left(D / r_{c c^{\prime}}\right)^{12}-\left(D / r_{c c^{\prime}}\right)^{6}\right] .
$$

The results of calculations for phase transitions at constant density using the above model potential with $n_{\mathrm{s}}=9$ and $L / D=5$ are shown in figure 6 . They correspond to two values of the ratio $\beta_{\mathrm{d}} / \beta_{\mathrm{L}-\mathrm{J}}$ that measures the strength of the dipolar interaction relative to the L-J potential. The obvious difference from the respective phase diagrams of figures $3(a)$ and 5 is that now the high temperature phase is always the isotropic one. The inverse temperature $\left(\beta_{\mathrm{d}}\right)_{\mathrm{N}-\mathrm{I}}$ at the transition is a single valued function of the density for any value of the eccentricity. Accordingly, the high temperature re-entrant nematic phase for off-centre dipoles is not obtained in this case. At sufficiently large $\beta_{\mathrm{d}}$ the shape of the phase diagrams and their trend of variation with $x$ are similar to those obtained in figures $3(a)$ and 5 for the spherocylinders.

\section{Summary and discussion}

We have used the variational cluster expansion method to investigate the effect of electric dipole interactions on the nematic ordering of uniaxial rod like molecules. The free energy was formulated in terms of two-molecule cluster contributions, yielding the lowest order approximation that contains the intermolecular potential 
explicitly. Three alternative molecular models were considered for the non-polar part of the intermolecular interaction, namely, hard spherocylinders with and without attractions, and rigid Lennard-Jones arrays. The electric dipoles were positioned along the molecular axis of cylindrical symmetry at various distances from the molecular centre.

The results show that the thermodynamically stable nematic phase is non-polar (in the absence of external fields) and that the conditions of its coexistence with the isotropic phase are, in general, influenced weakly by the dipolar interactions. Perhaps the most interesting feature of the results is the dependence of the stability of the nematic phase on the position of the dipole along the molecular axis. The dipolar eccentricity is predicted to affect substantially the nematic-isotropic transition temperature and the average dipole-dipole interaction energy, but to have marginal effects on the order parameter $\left\langle P_{2}\right\rangle$, on the compressibility, and on the Kirkwood $g$ factors. An important qualitative difference between central and off-centre dipoles is that the latter can give rise to nematic phase re-entrance. The conditions for re-entrance are, however, not determined exclusively by the strength and the position of the dipole; they are to some extent dependent on the non-polar part of the intermolecular interaction in the sense that soft repulsions and strong attractions are shown to inhibit re-entrance.

In spite of the severe approximations employed in the calculations, the results are in qualitative agreement with available Monte Carlo simulation results on spherocylinders with central dipoles. The essential features obtained from these simulations [13] for the role of dipole moments (slight effects on the order parameter $\left\langle P_{2}\right\rangle$ and the pressure, promotion of substantial antiparallel molecular association) also emerge from the present calculations. In addition, some of the subtleties of dipolar effects are revealed and a mechanism of phase re-entrance emerges naturally. Nevertheless, the theory, in the form presented here, is too simple to warrant a quantitative comparison with experimental results on real or simulated liquid crystals, or even to account qualitatively for the multitude of phases that can be formed by elongated polar molecules. This is primarily due to the following two limitations:

(a) The chosen form of the variational field corresponds to spatially uniform phases and therefore allows only for isotropic and nematic ordering. Thus, for example, the destabilization of the nematic phase with decreasing temperature, observed for off-centre dipoles, is necessarily manifested as a transition to the isotropic phase simply because no other phase is available. Clearly a thorough study of the phase diagram requires the extension of the variational field to allow for a wider variety of phases, in particular for smectics which, as suggested by computer simulations [12], are the favoured mode of ordering for rods with off centre dipoles.

(b) In fluids, the restriction to second order clusters is equivalent to retaining up to linear terms in a density expansion (see equation (21)) and therefore constitutes a major source of inaccuracy at liquid crystal densities $[25,30,35,36]$. The third and fourth order clusters have been shown to bring about substantial improvement in the description of orientable molecules on a lattice [27]. Work in progress indicates that, for fluids, the inclusion of third order clusters (which is not equivalent [29] to the third order viral extensions $[30,35,36]$ of the Onsager theory), improves considerably the agreement with $\mathrm{MC}$ results, albeit at a considerable computational cost. 
This work was supported in part by the EC under Human Capital and Mobility Programme contract ERBCHRXCT930161.

\section{References}

[1] Born, M., 1916, Sits. Phys. Math., 25, 614; 1918, Ann. Phys., 55, 221.

[2] Frenkel, D., Lekkerkerker, H. N. W., and Stroobants, A., 1988, Nature, 332, 822.

[3] Goodby, J. W., 1986, Science, 231, 350.

[4] Hardouin, F., Levelut, A. M., Achard, M. F., and Sigaud, G., 1983, J. Chim. Phys., 80, 53.

[5] DE JeU, W. H., 1983, Phil. Trans. R. Soc. Lond., A, 309, 217.

[6] Dunmur, D. A., and Toriyama, K., 1986, Liquid Crystals, 1, 169.

[7] Cladis, P. E., 1988, Molec. Crystals liquid Crystals, 165, 85.

[8] Wei, D., Patey, G. N., and Perera, A., 1993, Phys. Rev. E, 47, 506.

[9] Netz, R. R., and Berker, A. N., 1992, Phys. Rev. Lett., 68, 333.

[10] Wei, D., and Patey, G. N., 1992, Phys. Rev. Lett., 68, 2043.

[11] Weis, J. J., Levesque, D., and ZarragoicoecheA, G. J., 1992, Phys. Rev. Lett., 69, 913.

[12] Levesque, D., Weis, J. J., and Zarragoicoechea, G. J., 1993, Phys. Rev. E, 47, 496.

[13] Weis, J. J., Levesque, D., and Zarragoicoechea, G. J., 1993, Molec. Phys., 80, 1005.

[14] Kusalik, P. G., 1994, Molec. Phys., 81, 199.

[15] Palffy-Muhoray, P., Lee, M. A., and Petschek, R. G., 1988, Phys. Rev. Lett., 60, 2303.

[16] Baus, M., and Colot, J. L., 1989, Phys. Rev. A, 40, 5444.

[17] Terzis, A. F., and Photinos, D. J., 1994, Molec. Phys., 83, 847.

[18] Photinos, D. J., Poon, C. D., Samulski, E. T., and Toriumi, H., 1992, J. phys. Chem., 96, 8176.

[19] Photinos, D. J., and Samulski, E. T., 1993, J. chem. Phys., 98, 10009.

[20] Strieb, B., Callen, H. B., and Horwitz, G., 1963, Phys. Rev., 130, 1798.

[21] Dowel, F., 1985, Phys. Rev. A, 31, 3214.

[22] Perera, A., and Patey, G. N., 1989, J. chem. Phys., 91, 3045.

[23] Parsons, J. D., 1979, Phys. Rev. A, 19, 1225.

[24] OnSager, L., 1949, Ann. N. Y. Acad. Sci., 51, 627.

[25] Vroege, G. J., and Lekkerkerker, H. N. W., 1992, Rep. Prog. Phys., 55, 1241.

[26] Ypma, J. G. L., Vertogen, G., and Koster, H. T., 1976, Molec. Crystals liquid Crystals, $37,57$.

[27] Van der Haegen, R., Debruyne, J., Luyckx, R., and Lekkerkerker, H. N. W., 1980, J. chem. Phys., 73, 2469.

[28] Chicoli, C., Pasini, P., Biscarini, F., and Zannoni, C., 1988, Molec. Phys., 65, 1505.

[29] Manuscript in preparation.

[30] Turpto-Margo, B., and Evans, G. T., 1990, J. chem. Phys., 93, 4254.

[31] Luckhurst, G. R., 1979, The Molecular Physics of Liquid Crystals, edited by G. R. Luckhurst and G. W. Gray (New York: Academic).

[32] Vertogen, G., and DE Jeu, W. H., 1989, Thermotropic Liquid Crystals, Fundamentals (Heidelberg: Springer).

[33] Sear, R. P., and Jackson, G., 1994, Molec. Phys., 82, 473.

[34] Dunmur, D. A., and Palffy-Muhoray, P., 1992, Molec. Phys., 76, 1015.

[35] Tuipto-Margo, B., and Evans, G. T., 1991, Molec. Phys., 74, 85.

[36] Samborski, A., Evans, G. T., Mason, C. P., and Allen, M. P., 1994, Molec. Phys., 81, 263. 